\title{
CHARACTERISTICS OF PNEUMATIC TUNERS OF TORSIONAL OSCILLATION AS A RESULT OF PATENT ACTIVITY
}

\author{
Jaroslav HOMIŠIN* \\ *Faculty of Mechanical Engineering, Department of design, Automotive and Transport Engineering, Technical University of Košice, \\ Letná 9, 04001 Košice, Slovakia \\ jaroslav.homisin@tuke.sk
}

received 4 April 2016, revised 12 December 2016, accepted 13 December 2016

\begin{abstract}
Mechanical systems with combustion engines, compressors, pumps and fans, can be characterized as torsional oscillating mechanical systems (TOMS). It is therefore necessary to control their dangerous torsional vibrations. It was confirmed that dangerous torsional vibration can be reduced to acceptable level by an appropriate adjustment, respectively by tuning the TOMS. According to several authors, the most appropriate way of system tuning is application of suitable flexible element, which is flexible shaft coupling. It turned out that one of the types of shaft couplings, which are particularly suited to meeting this objective are pneumatic flexible shaft couplings, to act as so-called pneumatic tuners of torsional oscillations. The issue of research and development of pneumatic tuners of torsional oscillations, among other things is, long-term in the focus of the author. The existence of tuners creates the opportunity to develop new ways of tuning torsional oscillating mechanical systems. The author of the scientific article will focus on the characteristics of developed pneumatic tuners of torsional oscillation in terms of their design, construction, function, significance advantages and conditions imposed on pneumatic tuners based on the results of his patent activity. Simultaneously provides information about the characteristic properties of pneumatic tuners of torsional oscillations in the general design.
\end{abstract}

Key words: Pneumatic Tuner of Torsional Oscillation, Torsional Oscillating Mechanical System, Characteristics of Pneumatic Tuners

\section{INTRODUCTION}

In mechanical systems with reciprocating machines as driving or driven devices dangerous torsional vibration occurs. It means that Mechanical systems with combustion engines, compressors, pumps and fans, can be characterized as torsional oscillating mechanical systems (TOMS).

TOMS can be considered, from dynamic point of view, as systems working most frequently in supercritical range with relatively quick transient process by startup and braking. In terms of regulation they can be categorized as a controlled system with incomplete information. Incomplete information is reflected particularly in the area of unexpected (random) of failure effects. The most common effects of random disturbances in TOMS include the effects piston machines themselves. On this basis, it can be stated that especially piston machines introduce into these systems increased torsional vibration causing their excessive dynamic load.

Therefore, it is necessary to control their dangerous torsional vibration. It was confirmed that dangerous torsional vibration can be changed on the acceptable level by an appropriate adjustment, respectively tuning the TOMS. Under tuning the mechanical system should be understood an appropriate adjustments of dynamic properties of some of its members to the dynamics of the system. This means that to achieve the aims to reduce unacceptable torsional vibration, it is necessary that any TOMS has to be previously tuned.

According to several authors, the most preferred method of system tuning is the application of a suitable elastic element, which is considered a flexible shaft coupling (Ankarali et al., 2012; Bolek et al., 1967; Gao et al., 2010; Grega et al., 2015; Homišin, 1996; Homišin, 2008; Yubao, 2013; Lacko, 1971; Pol'akov, 1979; Singiresu, 1996; Timošenko, 1960; Wilson, 1968).

With this issue any manufacturer of flexible shaft couplings is seriously concerned. The world's leading manufacturers of flexible couplings are currently trying to solve this problem by using flexible shaft couplings with particularly low torsional rigidity (Bingzhao et al., 2010; Böhmer, 1983; Curà et al., 2013; Gao et al., 2010; Homišin, 1986; Homišin, 2002; Homišin, 2016; James et al., 2014; Lunke et al., 1983; Zoul, 1988; Zoul, 1989).

It was shown that one of the types of shaft couplings, which are particularly suited to achieve this are the pneumatic flexible shaft couplings, which acts as a so-called pneumatic tuners of torsional oscillations.

The issue of research and development of pneumatic flexible shaft couplings is, among other things, long in the focus of the author. In the scientific research field the author focuses on proper design of new types of shaft couplings, and pneumatic flexible shaft couplings (newly developed types of flexible shaft couplings are resulting from the claims of more than seventy granted patents of the author (Homišin, 1986a, 1986b, 1995a, 1995b, 1996b, 1998, 2002a, 2016), investigation of the basic properties of developed pneumatic couplings (the author dealt with the research of pneumatic flexible shaft coupling in scientific papers (Homišin, 1996a; Grega et al., 2015).

Particularly he aims at facilitating the application of the given couplings in various types of torsional oscillating mechanical systems with the objective of optimal tuning, thus optimizing them 
in terms of achieving minimum levels of dangerous torsional oscillation, or its removal. The existence of pneumatic couplings, thus pneumatic tuners of torsional oscillations, creates the possibility of implementing new ways of torsional oscillating mechanical systems tuning. The aim of the scientific article is the focus on the characteristics of the pneumatic tuners of torsional oscillation developed by the author in terms of their structure, function, importance and advantages based on his patent activity, as well as presenting their characteristic properties in general terms.

\section{BRIEF CHARACTERISTICS FUNCTION AND IMPORTANCE OF FLEXIBLE SHAFT COUPLINGS}

Based on the general characteristics of flexible shaft couplings applies that in addition to compensating axial, radial and angular shaft misalignment serve as a very effective means of tuning of any TOMS in terms of torsional vibration size. Appropriate tuning of TOMS is ensured by an appropriate adaptation of the dynamic properties of flexible coupling to the dynamics of mechanical systems, based on a detailed dynamic analysis. It follows that a properly selected flexible shaft coupling is used to limit the formation of resonances in the operating speed range (OSR) and thus to control dangerous torsional vibrations. Usually moves the natural angular frequency of torsional oscillation $\Omega_{O}$, and thus the critical speed $n_{k} \rightarrow n_{k}{ }^{*}$ from individual load torque harmonic components (particularly from the major harmonic component), in the lower rotation frequency area (Fig. 1). As a result, by the rapid start-up, respectively braking no unacceptable torsional vibrations occur and are damped by the coupling.

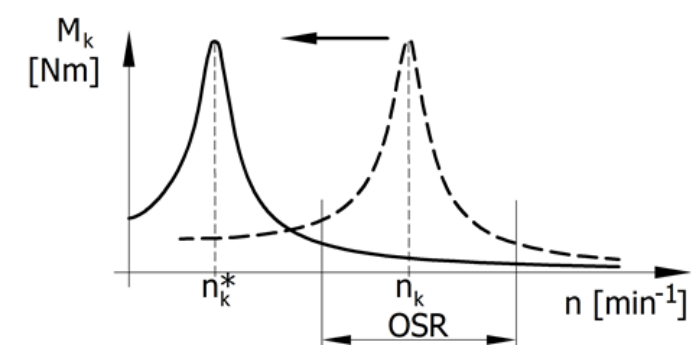

Fig. 1. Demonstration of the effect of flexible couplings on the dynamics of the system

With regard to their basic characteristics, i.e. dynamic torsional stiffness $\mathrm{k}$ and the damping coefficient $\mathrm{b}$, it should be noted that they are affected by material (metal, rubber, plastics), shape, number, size and design modifications to their flexible elements. Thus, they depend on various factors, each in its own way influences the characteristics of flexible couplings. Based on their impact the above factors can be divided into two groups - stable and unstable factors. The shape, number, size and various structural modifications of the elastic members can be categorized as stable factors. Material of elastic elements to a group of unstable factors, as they change its original characteristics as a result of fatigue and aging.

The above influences acting on the basic characteristics of shaft couplings show that each flexible coupling member, whether it is metallic or non-metallic, is exposed to fatigue or aging in the case of acting external forces. As a result, there is a change of coupling load characteristics $M_{k}=f(\varphi)$ (with respect to initial characteristics), and thus to change of its basic characteristic properties. It should also be noted that any currently used linear or non-linear coupling has only one characteristic. The result of this fact, it is only one course of natural speed frequency in the Campbell diagram. Change of the characteristics of the flexible coupling due to an appropriate adaptation of the dynamic properties to the dynamics of TOMS means using a different elastic element or using another flexible coupling. Taking into account this fact, as well as the instability of dynamic properties of flexible couplings caused by aging and fatigue, the elastic elements cause detuning of a previously tuned TOMS. In this case, the tuning member, i.e. flexible shaft coupling, has no possibility of eliminating or reducing dangerous increasing torsional vibration (Zoul, 1982).

Taking into account the above considerations in order of tuning or continuous tuning (Attention should be paid to the option of tuning torsional oscillating mechanical systems with pneumatic tuners out of service and continuous tuning of the system during operation at steady-state in the following article entitled: "New ways of tuning torsional oscillating mechanical systems".) of TOMS with intention of reducing dangerous torsional oscillations, we proposed to use pneumatic flexible shaft couplings developed by us i.e. pneumatic tuners of torsional oscillations in our understanding.

\section{CONDITIONS REQUIRED FROM PNEUMATIC TUNERS OF TORSIONAL OSCILLATION FOR THEIR APPLICATION IN MECHANICAL SYSTEMS}

Pneumatic tuners must in any TOMS meet the following requirements:

- Compensation of axial, radial and angular misalignments caused by manufacturing irregularities.

By the load torque transfer the compensation of axial, radial and angular misalignments and also the angular twist of driven to driving part of pneumatic tuner, is ensured by its flexible compression space).

- Ensuring the stable dynamic properties and stable load torque transfer during lifetime of mechanical system.

By twisting the pneumatic tuner the compression of gaseous medium proportional to load is achieved, thus implementing flexible load torque transfer in TOMS. Stable flexible transfer is ensured by the use of gaseous medium (in this case air) as flexible material in the tuner, which has a dominant influence on pneumatic tuner basic characteristics, and throughout its lifetime it is not subject to aging or fatigue (Krejčír, 1986). Consequently, the pneumatic tuner does not lose its initial characteristics, indicating its stability for the lifetime of TOMS. Pneumatic tuners of torsional oscillations have not one, but a range of load characteristics and also the range of characteristic properties within the range of gaseous medium pressure $p$ in the compression space. Characteristic properties of these tuners are affected by change of gaseous medium pressure $p$ - specifically by tangential, differential and axial tuners - and also by constant twist angle $\varphi_{k}$ selection with corresponding gaseous medium pressure $p$ change - specifically by pneumatic tuner of torsional oscillation with autoregulation).

- Ability of appropriate tuning of torsional oscillating mechanical systems, thus adapting the dynamic properties to the dynamic properties of the system.

Based on change of gaseous medium pressure $p$ in the compression space of pneumatic tuner we are changing, i.e. tuning the torsional stiffness $\mathrm{k}$, which has a decisive influence 
on the natural frequency of the system $\Omega_{O}$, where $I_{\text {red }}$ is the reduced mass moment of inertia of the system:

$$
\Omega_{O}=\sqrt{k / I_{\text {red }}} .
$$

Based on above it follows the principle of appropriate tuning of TOMS by pneumatic tuners of torsional oscillation, which essential nature is to adapt the natural angular frequency of the system $\Omega_{O}$ to the exciting angular frequency $\omega$ during operation of the given system so, that in the operating range of the system resonance $\left(\Omega_{O}=\omega\right)$ and dangerous torsional vibration does not occur).

\subsection{Tangential pneumatic tuners of torsional oscillation}

Tangential pneumatic tuners of torsional oscillation (Figs. $2-7$ ) consist of driving (1) and driven part (2), between them is located the compression space. The compression space is formed by pneumatic-flexible elements tangentially spaced around the circumference (3). Pneumatic-flexible element is characterized by the rubber-cord single or double bellows filled with gaseous medium. When transferring the load torque there are two pneumatic-flexible elements simultaneously compressed and two expanded, ensuring design of two-way coupling (Figs. 2, 4, 5 and 7). If the compression space is formed by three pneumatic-flexible elements (3), a design of one-way pneumatic coupling is created (Figs. 2 and 5). Through valve (4) the filling of compression space with gaseous medium is realized. The design of pneumatic coupling allows the interconnection of individual pneumaticflexible elements with changeable throttle nozzles (5) and hoses (6). This created three basic alternatives of interconnection (A, B,

C) of pneumatic coupling:

- pneumatic tuner of torsional oscillation, without interconnection of pneumatic flexible elements, with type designation: 4-1/XXX-T-A (Fig. 2) (where the meaning of labeling is following: 4 - number of elements, 1 - single bellows pneumatic-flexible elements with outer diameter $\mathrm{XXX} \mathrm{mm}, \mathrm{T}-$ with tangential distribution, and $\mathrm{A}$ - without interconnection), and 3-1/XXX-T-A (Fig. 3),

- pneumatic tuner of torsional oscillation with mutual interconnection of pneumatic-flexible elements, type designation: 4-1/XXX-T-B (Fig.4),

- pneumatic tuner of torsional oscillation with full mutual interconnection of pneumatic-flexible elements, type designation: 4-1/XXX-T-C (Fig. 5) and 3-1/XXX-T- C (Fig. $6)$. a)

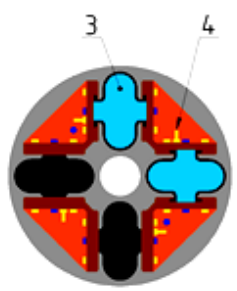

b)

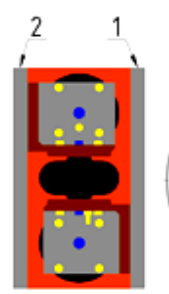

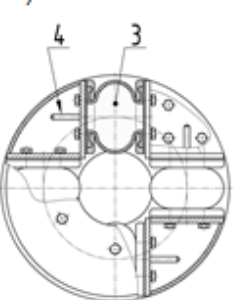

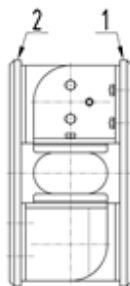

Fig. 2. Tangential pneumatic tuner without pneumatic-flexible elements interconnection: a) graphic representation, b) design representation a)

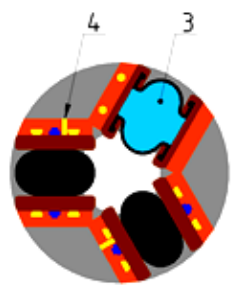

b)

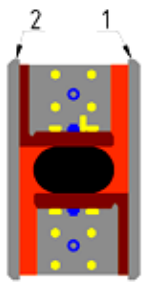

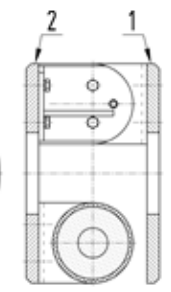

Fig. 3. Tangential pneumatic tuner without pneumatic-flexible elements interconnection: a) graphic representation, b) design representation

a)

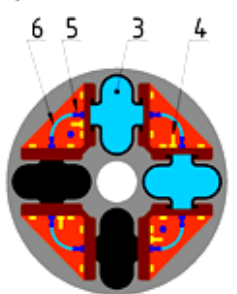

b)

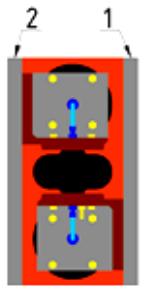

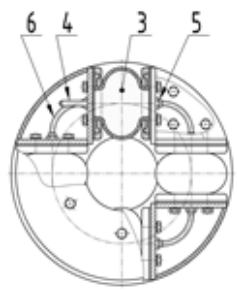

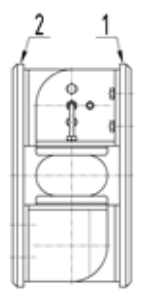

Fig. 4. Tangential pneumatic tuner with mutual interconnection of pneumatic-flexible elements: a) graphic representation, b) design representation

a)

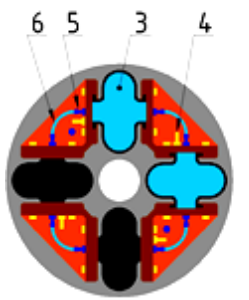

b)

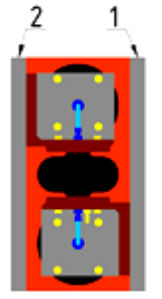

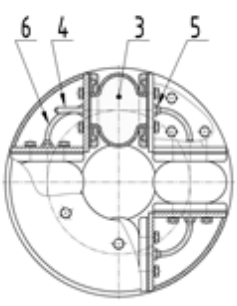

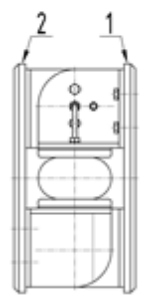

Fig. 5. Tangential pneumatic tuner with full mutual interconnection of pneumatic-flexible elements: a) graphic representation, b) design representation

a)

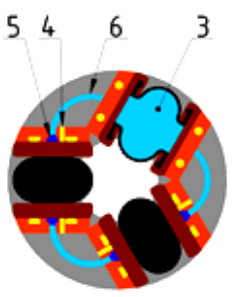

b)

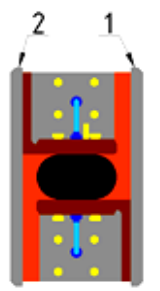

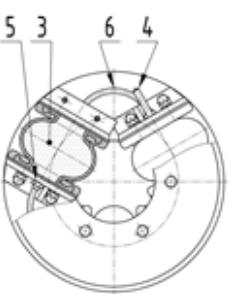

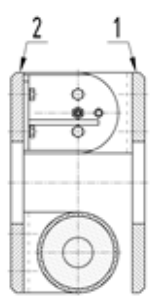

Fig. 6. Tangential pneumatic tuner with full mutual interconnection of pneumatic-flexible elements: a) graphic representation, b) design representation

a)

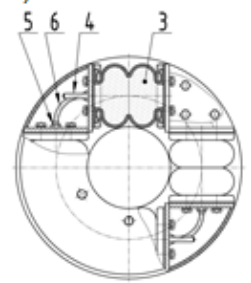

b)

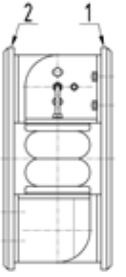

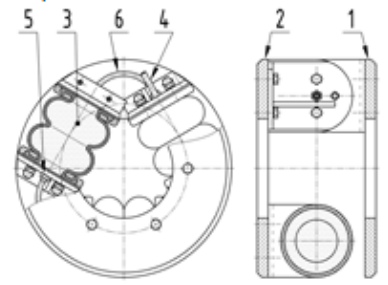

Fig. 7. Tangential pneumatic tuner with full mutual interconnection of pneumatic elements: a) type 4-2/XXX-T-C, b) type 3-2/XXX-T-C 
In Fig. 7 pneumatic tuners of torsional oscillation with full mutual interconnection of double-bellows pneumatic-flexible elements type 4-2/XXX-T-C and type 3-2/XXX-T-C are presented.

\subsection{Differential pneumatic tuners of torsional oscillation}

Differential pneumatic tuners of torsional oscillation type 3-1/XXX-D-C (3 - three, 1 - single-bellows pneumatic flexible elements with outer diameter - XXX mm, with differential elements $-D$ with interconnection - C) (Fig. 8 a, b) or 3-2/XXXD-C (3 - three, 2 - double-bellows pneumatic flexible elements with outer diameter - XXX mm, with differential elements $-D$ a with interconnection - C) (Fig. $9 \mathrm{a}, \mathrm{b}$ ) consists of the driving part (1) and the driven part (2), between them is a compression space filled with a gaseous medium (in our case air). Compression space consists of three circumferentially spaced and interconnected differential elements. Each differential element consists of compressed (3) and expanded pneumatic-flexible element (4). Interconnection of differential elements is provided by interconnecting hose (5). Through the valve (6), the filling of compression space of coupling, thereby changing the gaseous media pressure is ensured. a)

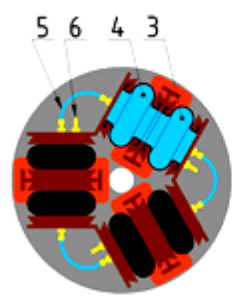

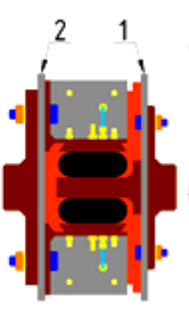

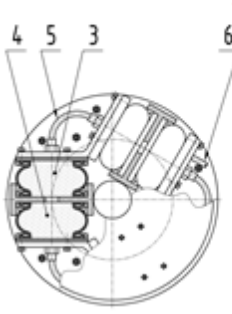

b)

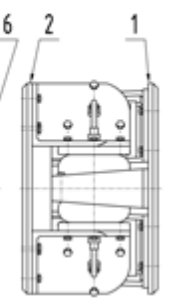

Fig. 8. Differential pneumatic tuner with full mutual interconnection of pneumatic-flexible elements type 3-1/XXX -D-C: a) graphic representation, b) design representation a)

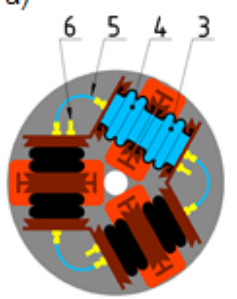

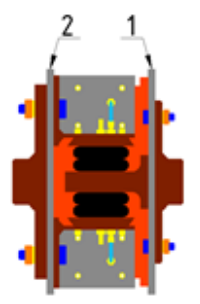

b)

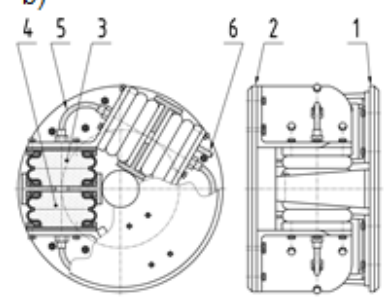

Fig. 9. Differential pneumatic tuner with full mutual interconnection of pneumatic-flexible elements type 3-2/XXX-D-C: a) graphic representation, b) design representation

\subsection{Pneumatic tuners of torsional oscillation with autoregulation}

Differential pneumatic tuners of torsional oscillation with autoregulation (Fig. 10), which basic principle results from patent claims of granted patents (Homišin, 1995; 1996), have a common design basis. The main difference consists in regulator (6) which enables to keep constant angle of twist $\varphi_{\mathrm{k}}$. The basic principle of the PTOTO is the self-regulation ability of the angular twisting, caused due to actual change of loading torque, into the given constant angular value $\varphi_{k}$. This ensures the self-regulation of the gaseous medium pressure $p$ in the compression space of tuner, thus adapting it to the current value of the load torque. This selfregulation of gaseous medium pressure in the compression space in the tuner has an immediate influence onto the characteristic of pneumatic tuner (Figs. 14 and 15) and, of course, onto the torsional stiffness $k$ (Figs. $11 \mathrm{a}, \mathrm{b}$ ), in this way it can be changed the natural frequency of the system $\Omega_{O}$.

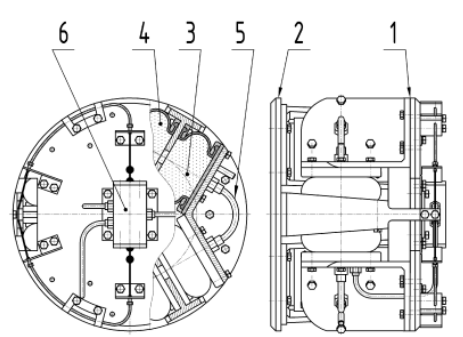

Fig. 10. Pneumatic tuner of torsional oscillation with autoregulation

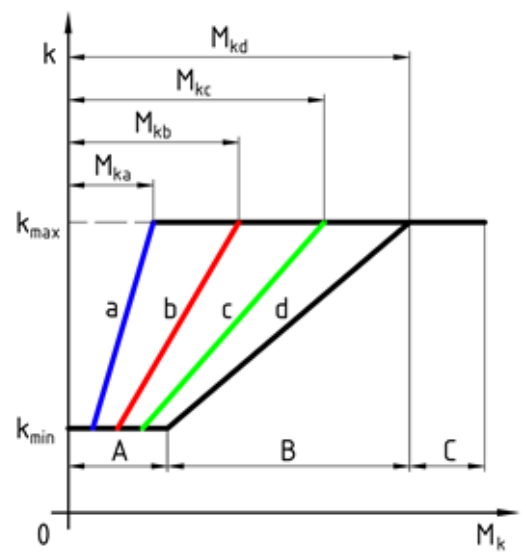

Fig. 11. Courses of torsional stiffness $k$ of pneumatic tuner of torsional oscillation with autoregulation depending on load torque $M_{k}$

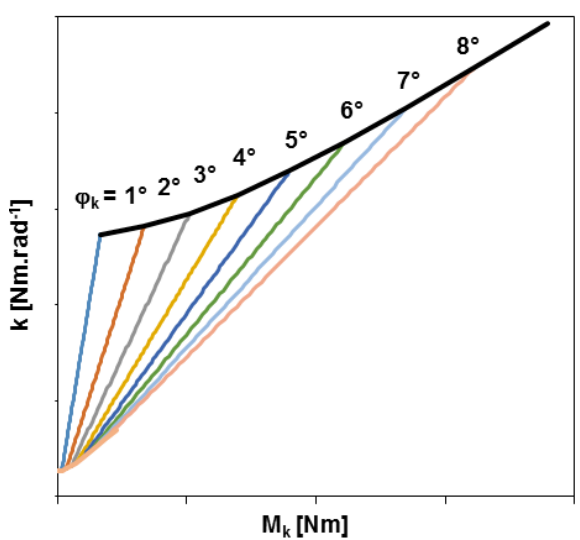

Fig. 12. Courses of torsional stiffness $k$ of pneumatic tuner of torsional oscillation with autoregulation, type 3-1/XXX -D-C depending, on load torque $M_{k}$

In Fig. 11 and 12 is schematically presented a behaviour of torsional stiffness $k$ of a PTOTO in dependence on loading torque $M_{k}$. To each of the calculated constant angles of twist $\varphi_{\mathrm{ka}}, \varphi_{\mathrm{kb}}, \varphi_{\mathrm{kc}}$ and $\varphi_{\mathrm{kd}}$ belongs one behaviour of torsional stiffness $a, b, c, d$. The above-mentioned behaviours are limited with minimum and maximum values of torsional stiffness $k_{\min }$ and $\mathrm{k}_{\max }$ according to the pressures of gaseous medium from interval 
pmin and pmax in compression space of the PToTO. There are also presented behaviours illustrated by a fractional line, which consists of three areas: pre-regulation $-A$, regulation $-B$ and over-regulation $-C$ area. From this illustration it is evident that change of $\varphi_{\mathrm{ki}}$ influences interval of pre-regulation and regulation area, but it influences mainly the value of pneumatic tuner torsional stiffness during operational regime of the system. There are also influenced values of loading torque $M_{k a}, M_{k b}, M_{k c}$ and $M_{k d}$, with regard to the maximum value $k_{\max }$ of torsional stiffness. The pneumatic tuner with an increasing value of constant angle of twist, during a certain loading torque, has a declining torsional stiffness. In the case of the PTOTO with the maximum angle of twist value $\varphi_{\text {kmax }}$, from the relatively hard pneumatic coupling (behaviour a) becomes a high flexible pneumatic coupling (behaviour d), which is able to operate with considerably higher value of loading torque $M_{k}$ at maximum value of torsional stiffness.

\subsection{Axial pneumatic tuners of torsional oscillation}

The essence of axial pneumatic tuners design is the fact that between the drive hub (1) and driven hub (3) is located a floating body (2) solidly coupled with compression space of tuner formed by an elastic hollow body (4) and filled by gaseous medium (Fig. 13) (Homišin, 1986).

a)

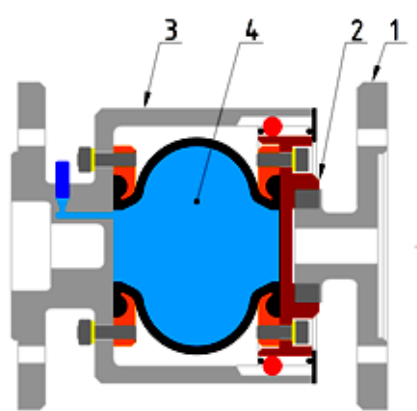

b)

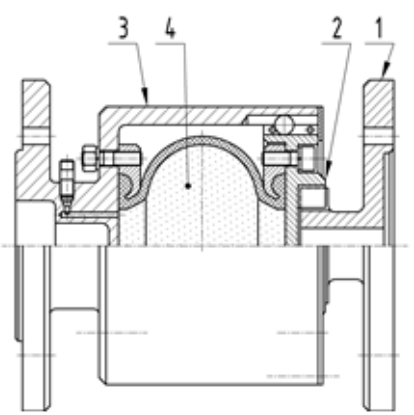

Fig. 13. Axial pneumatic tuner of torsional oscillation:

a) graphic representation, b) design representation

a)

b)

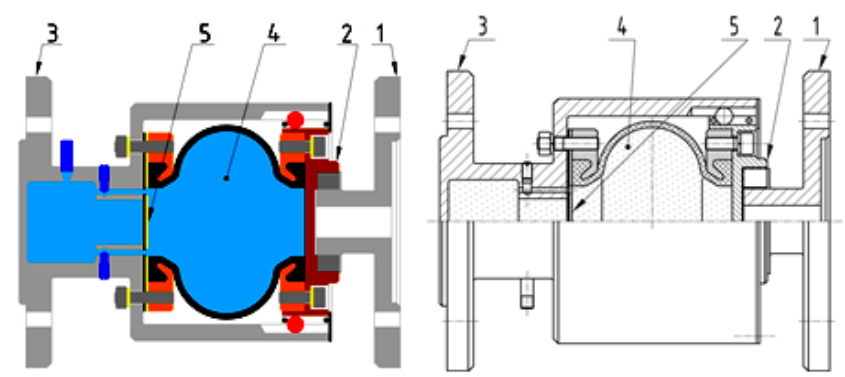

Fig. 14. Axial pneumatic tuner of torsional oscillation with damping: a) graphic representation, b) design representation

In Fig. 14 an axial tuner of torsional oscillation with damping is displayed (Homišin, 1986). From the nature of design of the pneumatic tuner follows that between the driving hub (1) and driven hub (3) is formed a compression space. Compression space filled with gaseous medium is divided by a membrane with throttle openings (5) divided into deformable and stationary part.
Deformable part of compression space if formed by a rubber element (4) membrane (5) separated from stationary part of compression space formed by chamber in part (3).

If the compression space of pneumatic tuner (Figs. 13 and 14 ) is filled by gaseous media with initial pressure $p_{1}$, it holds the floating body (2) in basic position, as we can see on the figure. By increasing of load torque in direction of thread teeth helix pitch of meshing parts (1) and (2) the axial shift of floating body (2) occurs, creating a compression of the gaseous medium in the coupling compression space. In the case of oscillating load torque transmission pulsation of carrier fluid occurs in the tuner compression space. Consequently, in proportion to the vibration torque the gaseous medium flows via throttle openings in membrane (5). The flow of gaseous medium is accompanied by throttling at a flow, which is characterized by throttling work in proportion to damping performance that is constant for pneumatic flexible shaft couplings with damping throughout its lifetime.

\section{PRESENTATION OF BASIC CHARACTERISTICS OF PNEUMATIC TUNERS OF TORSIONAL OSCILLATION}

Within the realized experiments, static and dynamic measurements of pneumatic tuners of torsional oscillation were performed.

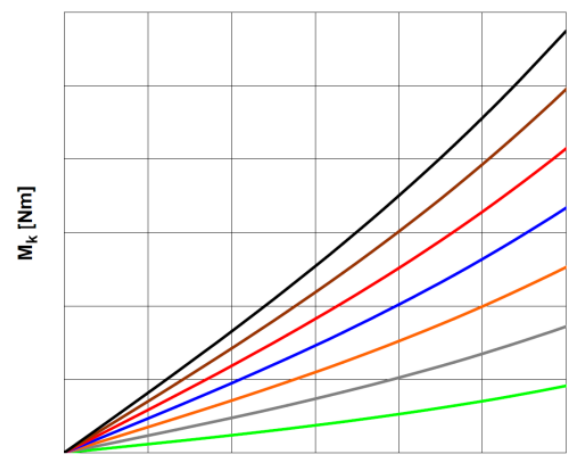

$\varphi\left[{ }^{\circ}\right.$

Fig. 15. Characteristics of pneumatic tuners of torsional oscillations in the pressure range $p=100 \div 700 \mathrm{kPa}$ type $4-1 / \mathrm{XXX}-\mathrm{T}-\mathrm{C}$, eventually $4-2 / X X X-T-C$ or $3-1 / X X X-D-C$, eventually $3-2 / X X X-D-C$

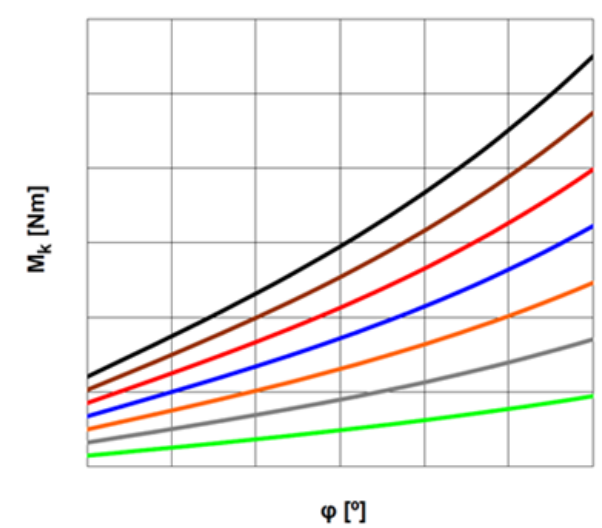

Fig. 16. Characteristics of pneumatic tuners of torsional oscillations in the pressure range $p=100 \div 700 \mathrm{kPa}$ type $3-1 / \mathrm{XXX}-\mathrm{T}-\mathrm{C}$, eventually $3-2 / X X X-T-C$ 
To illustrate the basic characteristics of pneumatic tuners of torsional oscillation only some of the measurement results are mentioned.

Obtained results of static and dynamic measurements showed that changing the pressure of the gaseous medium, pneumatic tuner is capable of working with always a different characteristic (Figs. 15 and 16), thus it is always able to work with other characteristic properties (torsional stiffness and damping coefficient).

Based on Figs. 15 and 16 it is possible to say that the characteristics of the pneumatic tuners are slightly nonlinear. Their course can be expressed by the equation (2) for Fig. 15 and equation (3) for Fig. 16:

$$
\begin{aligned}
& M_{S}=a_{1} \varphi+a_{3} \varphi^{3}, \\
& M_{S}=a_{0}+a_{1} \varphi+a_{3} \varphi^{3},
\end{aligned}
$$

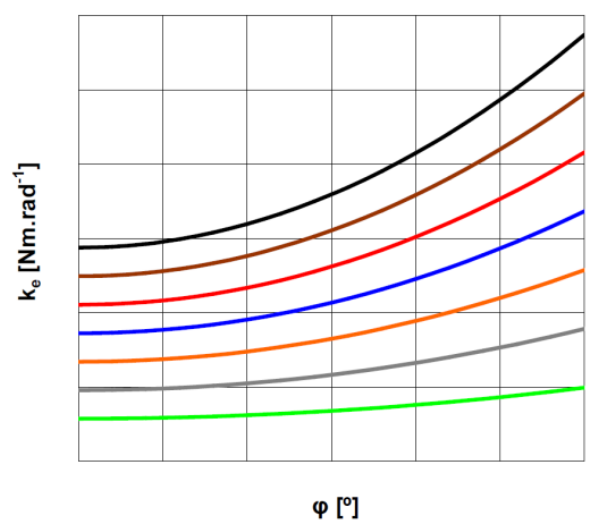

Fig. 17. Courses of equivalent torsional stiffness $k_{e}$ in pressure range $p=100 \div 700 \mathrm{kPa}$ depending on twist angle $\varphi$ of pneumatic tuner for the presented types of tuners

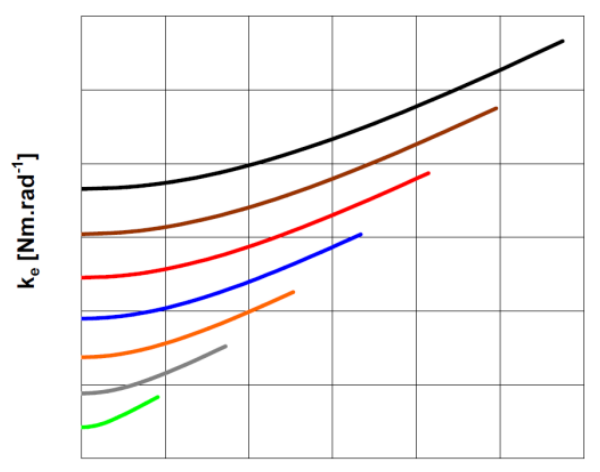

$\mathrm{M}_{\mathrm{k}}[\mathrm{Nm}]$

Fig. 18. Courses of equivalent torsional stiffness $k_{e}$ in pressure range $p=100 \div 700 \mathrm{kPa}$ depending of trasmitted load torque $\mathrm{M}_{\mathrm{k}}$ of pneumatic tuner for the presented types of tuners

From the characteristics of the air tuners we determine the courses of equivalent torsional stiffness of tuners $\mathrm{k}_{\mathrm{e}}$ with equivalent linearization method, according to (4) shown in Fig. 17:

$\mathrm{k}_{\mathrm{e}}=a_{1}+\frac{3}{4} a_{3} \varphi^{2}$.

Fig. 18 represents the range of equivalent torsional stiffness $\mathrm{k}_{\mathrm{e}}$ depending on the transmitted load torque $M_{k}$ of pneumatic tuners and also it gives us information on whether the tuner is suitable for work in a particular mechanical system.

By changing the pressure of the gaseous medium in the pneumatic tuners we largely influence on the size of their nonlinearity $\varepsilon=\mathrm{a}_{3} / \mathrm{a}_{0}$. Based on calculation it can be concluded that with the increase of pressure $100 \mathrm{kPa}$ to $700 \mathrm{kPa}$ nonlinearity coefficient decreases within the range $\varepsilon=15 \div 1,2$ (Fig. 19). The above results lead to the conclusion that in the range of the pressure of the gaseous medium $\mathrm{p}=200 \div 700 \mathrm{kPa}$ pneumatic tuner can be characterized as linear (That claim is documented among research report (Pešík et al., 1997; Zoul, 1982), in which the authors state that in the case of $\varepsilon<10$ flexible coupling is considered linear).

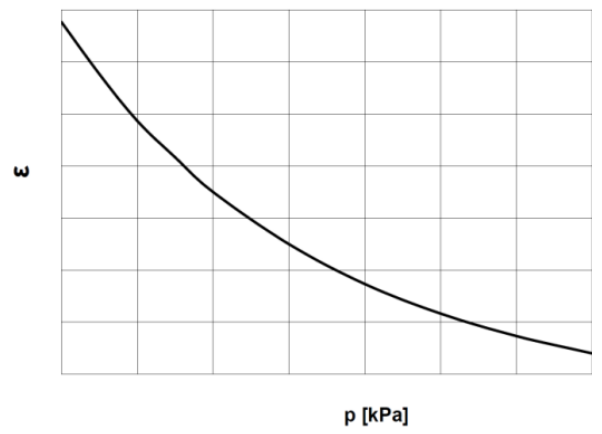

Fig. 19. Nonlinearity coefficient $\varepsilon$ courses depending on gaseous medium pressure $p$ in pneumatic tuners

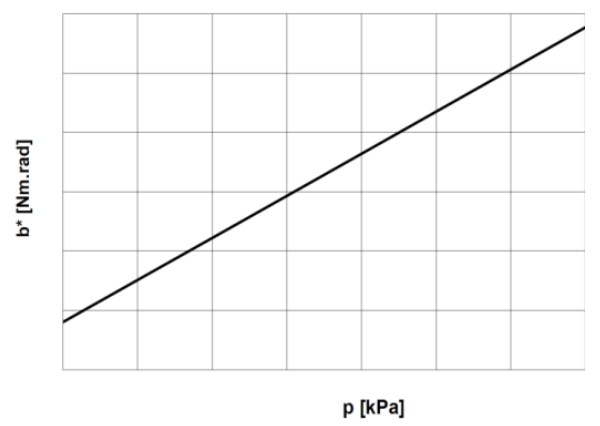

Fig. 20. Course of damping coefficient $b^{*}$ depending on gaseous medium pressure $p$ in pneumatic tuners

Further processing of measured values records determined equivalent damping coefficient $b_{e}$ of pneumatic tuners by individual pressures (with determining of equivalent coefficient of damping values be and damping coefficient $b^{*}$ values based on dynamic measurement by free oscillations the author deals in his candidate dissertation thesis (Homišin, 1989).). The results of our measurements confirmed the fact that the value of damping coefficient pneumatic tuners depends less likely on the preload, amplitude, and temperature, but to a greater extent on the frequency of oscillation $\omega$. Taking this into account impact of the frequency on damping coefficient can be expressed by the relationship (4), while the coefficient of damping $b^{*}$ (Fig. 20) is for a given preload amplitude and temperature approximately constant, i.e.:

$b_{e}=b^{*} / \omega$

The main and essential part of the presented pneumatic tuners is their compression chamber, which is formed by compressed and expanded pneumatic-flexible elements (rubber-cord bellows), filled with gaseous medium. In this case, the characteristics of the pneumatic tuners will depend both on the impact of the rubber cord bellows and also the influence of gaseous medium - as its filling. 
From the above problem, the question arises, which has a dominant influence on the fundamental characteristics pneumatic tuners - gaseous medium or a rubber-cord container?

In examining the magnitude of two components on the change of the characteristics of the pneumatic tuners it is considered in the article, that the increase in magnitude of the gas medium is in the range of $Q_{V}=66.22 \% \div 89.83 \%$ (course $-\mathrm{a}$ ), while the declining trend influence of rubber cord container has a range $Q_{G}$ $=33.78 \% \div 10.17 \%$ (course $-b$ ) depending on gaseous medium pressure $p$ in pneumatic tuner (Fig. 21).

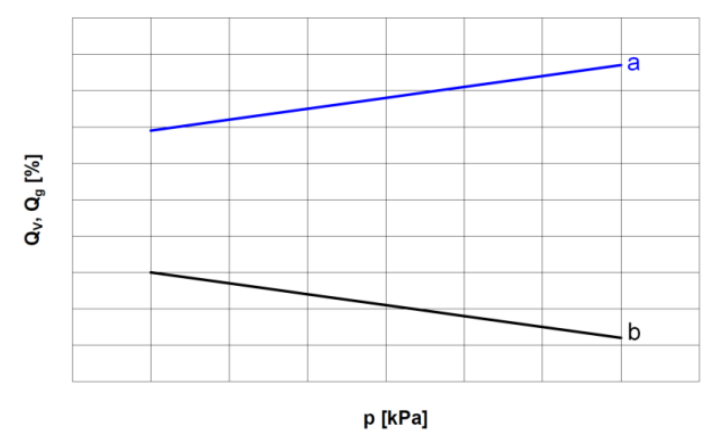

Fig. 21. Influence of gaseous medium $Q_{V}$ and rubber-cord container $Q_{G}$ depending on gaseous medium pressure $p$

On the basis of these values it can be stated that the effect of the gaseous medium at the beginning, i.e. by $p=100 \mathrm{kPa}$, is compared to the rubber-cord almost double, while on the end i.e. $p=700 \mathrm{kPa}$ shall take up to nine times the value.

From these results we can conclude that on the change of the basic characteristics of pneumatic tuners has a dominant influence definitely the gaseous medium.

\section{CONCLUSIONS}

Taking into account the above considerations in order to tuning respectively continuous tuning of TOMS and with the intention of restricting dangerous torsional oscillations, we propose to use pneumatic tuners of torsional oscillations developed by us. These pneumatic tuners have not one, but by a range of characteristics and also the range of the characteristics within the range of the pressure of the gaseous medium in the compression space. Characteristics of the tuners are influenced both by changing the pressure of the gaseous medium - specifically in tangential, differential and axial pneumatic tuners - and secondly, the couplings constant angle of twist while changing the pressure of the gaseous medium in them - namely in pneumatic tuners with autoregulation.

Based on the results of experimental verification, it was found that by changing the pressure of the gaseous medium in the compression space of the pneumatic tuner changes, i.e. tunes the dynamic torsional stiffness, which has a decisive influence on the natural frequency of the system. The essence of principle of tuning TOMS by pneumatic tuners is to adapt natural angular frequency of the mechanical system to the angular frequency of the excitation that in the range of the operation mode of the system to the resonance condition, and subsequently dangerous torsional vibration is avoided.

Pressure changes of the gaseous medium in the compression space of the pneumatic tuner can be done beyond or during oper- ation of mechanical systems

This resulted in two ways of tuning torsional oscillating mechanical systems suggested by us:

- Tuning of torsional oscillating mechanical systems out of operation,

- Tuning of torsional oscillating mechanical systems during operation in steady state.

\section{REFERENCES}

1. Ankarali A., Mecitoğlu Z., Diken H. (2012), Response spectrum of a coupled flexible shaft-flexible beam system for cycloidal input motion, Mechanism and Machine Theory, 47, 89-102.

2. Baworski A., Garbala K., Czech P., Witaszek K. (2015), Estimation of the Ability to Use a Mass of Air from a Moving Vehicle in Wind Turbine Propulsion, Scientific Journal of Silesian University of Technology, Series Transport, 88, 5-17.

3. Binglin L., Huajiang O., Wanyou L., Zhijun S. , Gang W. (2016), An indirect torsional vibration receptance measurement method for shaft structures, Journal of Sound and Vibration, 372, 11-30.

4. Bingzhao G., Hong C., Haiyan Z., and Kazushi S. (2010), A Reduced-Order Nonlinear Clutch Pressure Observer for Automatic Transmission, IEEE Transactions on Control Systems Technology. 18(2), $446-453$.

5. Böhmer J. (1983), Use of Vulkan Flexible Couplings with linear and progressive torsional load characteristic, MTZ, 44/5 (in German),

6. Bolek A., Krejčí V. (1967), Shaft Couplings. Praha, SNTL (in Czech).

7. Bulut G. (2014) Dynamic stability analysis of torsional vibrations of a shaft system connected by a Hooke's joint through a continuous system model, Journal of Sound and Vibration, 333(16), 3691-3701.

8. Curà F., Mura A. (2013), Experimental procedure for the evaluation of tooth stiffness in spline coupling including angular misalignment, Mechanical Systems and Signal Processing, 40, 545-555.

9. Czech P. (2012), Diagnosis of industrial gearboxes condition by vibration and time-frequency, scale-frequency, frequency-frequency analysis, Metalurgija, 51(4), 521-524

10. Czech P. (2013), Intelligent approach to valve clearance diagnostic in cars, Activities of Transport Telematics, 395, 384-391.

11. Czech P., Szyma B., Juzek M., Kałuża R. (2016), Braking process of sports motorbike, Acta Mechanica Slovaca, 20(2), 22-31.

12. El-Sayed A. T., Bauomy H. S. (2015), Passive and active controlers for suppressing the torsional vibration of multiple-degree-of-freedom system, Journal of Vibration and Control, 21(13), 2616-2632.

13. Folęga P., Wojnar G., Czech P. (2014), Influence of housing ribbing modification on frequencies and shapes of vibrations, Scientific Journal of Silesian University of Technology. Series Transport, 82, 81-86 (in Polish).

14. Gao W., Hao Z. (2010), Active control and simulation test study on torsional vibration of large turbo-generator rotor shaft, Mechanism and Machine Theory, 45, 1326-1336.

15. Genta G., Festini A., Delepine X. (2008), From Oil to magnetic fields: active and passive vibration control, Acta Mechanica et Automatica, 2(2), 11-20.

16. Grega R., Homišin J., Puškár M., Kul'ka J., Petróci J., Konečný B., Kršák B. (2015), The chances for reduction of vibrations in mechanical system with low-emission ships combustion engines, International Journal of Maritime Engineering, 157(A4), 235-240.

17. Homišin J. (1986a), Pneumatic flexible shaft coupling with damping, Patent No. 252034, (in Slovak).

18. Homišin J. (1986b), Pneumatic flexible shaft coupling, Patent No. 254180, (in Slovak).

19. Homišin J. (1995a), Pneumatic Flexible shaft coupling with autoregulation ability, Patent No. 278025, (in Slovak). 
20. Homišin J. (1996a), Contribution to a static optimalization of torsionaly oscillating mechanical systems, The shock and vibration digest, USA, 28/6, 86.

21. Homišin J. (1996b), Pneumatic coupling with auxiliary controller of constant twist angle, Patent No. 278272. (in Slovak).

22. Homišin J. (2002a), Axial pneumatic flexible shaft coupling, IPO SK Banská Bystrica, Patent № 275867, (in Slovak).

23. Homišin J. (2002b), New types of flexible shaft couplings, development - research - application, Vienala, Košice.

24. Homišin J. (2008), Tuning methods of torsional oscillating mechanical systems by pneumatic couplings, ATH, Bielsko-Biała, (In Polish).

25. Homišin J. (2016), Pneumatic flaxible axial shaft coupling, IPO SK Banská Bystrica, Patent No 288340, (in Slovak).

26. Homišin, J. (1995b), Shafft coupling with pneumatic-flexible units, IPO SK Banská Bystrica, Patent No 278024, (in Slovak).

27. Homišin, J. (1998), Pneumatic shaft coupling with flexible-damping units, IPO SK Banská Bystrica, Patent No 278750 (in Slovak).

28. James D., Van de Ven , Cusack J. (2014). Synthesis and baseline testing of a digital pulse-width-modulated clutch, Mechanism and Machine Theory, 78, 81-91.

29. Konieczny L. et al. (2015), Determination of the effect of tire stiffness on wheel accelerations by the forced vibration test method, Journal of Vibroengineering, 17(8), 4469-4477.

30. Krejčír O. (1986), Pneumatic vibroisolation - dissertation thesis, Liberec (in Czech).

31. Lacko P. (1971), Flexible shaft coupling as absorber of torsional vibrations, Strojírenství, 9, 71-74 (in Slovak).

32. Lazarz B., Wojnar G., Madej H., Czech P. (2009), Evaluation of gear power losses from experimental test data and analytical methods, Mechanika, 80(6), 56-63.

33. Łazarz B., Wojnar G., Czech P. (2011), Early fault detection of toothed gear in exploitation conditions, Maintenance and Reliability, 49(1), 68-77.

34. Lunke M., Beeftink G. B. (1983), Use of highly flexible couplings in energy-saving marine propulsion systems, Schiff und Hafen, 4/35, (in German).

35. Madej, H., Czech, P. (2009), Diagnostics of clearance in the pistoncylinder assembly using Hoelder coefficient, Diagnostyka, 1(49), 73-78.
36. Madej, H., Czech, P. (2009), Industrial gearboxes diagnosis by used higher order spectrum, Scientific Journal of Silesian University of Technology. Series Transport, 65, 51-56.

37. Pešík L., Němeček P. (1997), Identification of the dynamic system of a machine with an elastic base, McNU 97, Chicago, USA.

38. Polyakov V.S. (1979), Coupling guid, Leningrad, Mashinostrojenie, (in Russian).

39. Rosół M., Sapiński B. (2014), Autonomous Control System for a Squeeze Mode MR vibration isolator in an automotive engine mount, Acta Mechanica et Automatica, 8(3), 121-124.

40. Singiresu S. R. (1996), Engineering optimization theory and practice third edition, New York, John Wiley \& Sons, Inc.

41. Spruogis B., Turla V. (2006), A damper of torsional vibrations and an investigation of its efficiency, Strojniški vestnik - Journal of Mechanical Engineering, 52(4), 225-236.

42. Timoshenko, S. (1960), Vibration problems in engineering, Praha, SNTL (in Czech).

43. Wilson W.K. (1968), Practical solution of torsional vibration problems, volume 4, London, Chapman \& Hall LTD.

44. Wojnar G. (2009), Analysis of usefulness of different vibration signals for toothed gears diagnostics, Acta Mechanica et Automatica, 3(2), 111-114 (in Polish).

45. Yubao S., Jihong W., Dianlong Y. and Xisen W. ( 2013), Analysis and enhancement of torsional vibration stopbands in a periodic shaft system, Journal Of Physics D: Applied Physics, 46, 7-13.

46. Zoul V. (1982), Some aspects of flexible coupling development for sets with diesel engines, Strojirenství, 32(6/7), (in Czech)

47. Zoul V. (1988), Rato Highly Flexible Couplings, ČKD Praha, 20 (in Czech).

48. Zoul V. (1989) The use of flexible shaft couplings with low torsional stiffness for reducing dynamic torsional loads, IS ČKD Praha, 24-25, (in Czech).

Acknowledgement: This paper was written in the framework of Grant Project VEGA: "1/0688/12 - Research and application of universal regulation system in order to master the source of mechanical systems excitation". 